\title{
Letters
}

\section{Screening populations at low risk carries high risks}

EDITOR - In their key points Lu-Yao et al say that the lack of association between more intensive screening and treatment and lower mortality from prostate cancer means that trials should continue to settle this question. ${ }^{1}$ More research is the classic conclusion of all studies, but is it true?

Firstly, trials cannot be used to prove that something does not work. That is unethical; there has to be at least a sound presumption that screening will be effective. ${ }^{2}$ It is also futile, as newer screening tests will replace the older ones. The clamour for more testing will only increase.

Secondly, even if screening were effective, could it ever be efficient? The risk of any specific cancer is very low. The absolute risk of death from prostate cancer before the age of 75 was $1.33 \%$ in the Netherlands. ${ }^{-}$If we assume a risk reduction of $25 \%$ (which would be high), the absolute risk reduction is $0.33 \%$, which means that 300 men need to participate in a population screening programme to save one death.

If we take three to five screening rounds in the programme to obtain this reduction (which would be a small number), in 900-1500 cases participants will be at risk of overdiagnosis and overtreatment - known to cause impotence and incontinence - to save one death.

Cancer screening is based on biased information. Benefit is taken for granted but the risks and perils of screening are not mentioned. A simple calculation can easily be made for all screening procedures: numbers of screening procedures, numbers of diagnostic and therapeutic interventions induced, and numbers of disabilities caused by screening compared to numbers of lives saved. Many people will not believe you, which shows that they understand you all too well.

Part of informed consent is that people should be informed of the true, absolute risks, including risks of overdiagnosis and overtreatment. Cancer risk management should learn from cardiovascular risk management, ${ }^{4}$ where the risks are many times higher but only populations at increased risk are targeted.

Luc Bonneux, associate professor. Julius Center for Health Sciences, Utrecht Medical Center, HP D 01.335, PO Box 85500NL-3508 GA Utrecht, Netherlands l.bonneux@jc.azu.nl 
1. Lu-Yao G, Albertsen PC, Stanford JL, Stukel TA, Walker-Corkery ES, et al. Natural experiment examining impact of aggressive screening and treatment on prostate cancer mortality in two fixed cohorts from Seattle area and Connecticut. BMJ 2002; 325: 740[Abstract/Free Full Text]. (5 October.)

2. Adami HO, Baron JA, Rothman KJ. Ethics of a prostate cancer screening trial. Lancet 1994; 343: 958-960[ISI][Medline].

3. Netherlands Cancer Registry. Incidence of cancer in the Netherlands 1997. Amsterdam: Netherlands Cancer Registry, 2001.

4. Jackson R, Barham P, Bills J, Birch T, McLennan L, MacMahon S, et al. Management of raised blood pressure in New Zealand: a discussion document. BMJ 1993; 307: 107110[ISI][Medline].

(C) 2003 BMJ Publishing Group Ltd

\section{Related Article}

Natural experiment examining impact of aggressive screening and treatment on prostate cancer mortality in two fixed cohorts from Seattle area and Connecticut

Grace Lu-Yao, Peter C Albertsen, Janet L Stanford, Therese A Stukel, Elizabeth S Walker-Corkery, and Michael J Barry

BMJ 2002 325: 740. [Abstract] [Full Text] 